Santa Clara University

Scholar Commons

Physics

College of Arts \& Sciences

$10-3-2003$

\title{
Resistive transitions in quench-condensed Bi films near a normal-metal ground plane
}

Richard P. Barber Jr.

Santa Clara University, rbarber@scu.edu

Ben Kain

Follow this and additional works at: http://scholarcommons.scu.edu/physics

Part of the Physics Commons

\section{Recommended Citation}

B. Kain and R. P. Barber, Jr., "Resistive transitions in quench-condensed Bi films near a normal-metal ground plane", Physical Review B, 68, 134502, (2003).

This Article is brought to you for free and open access by the College of Arts \& Sciences at Scholar Commons. It has been accepted for inclusion in

Physics by an authorized administrator of Scholar Commons. For more information, please contact rscroggin@scu.edu. 


\title{
Resistive transitions in quench-condensed Bi films near a normal-metal ground plane
}

\author{
B. Kain* and R. P. Barber, Jr. \\ Department of Physics, Santa Clara University, Santa Clara, California 95053, USA \\ (Received 15 April 2003; revised manuscript received 29 May 2003; published 3 October 2003)
}

\begin{abstract}
We report observations of the zero-field resistive transitions of superconducting quench-condensed $\mathrm{Bi}_{0.97} \mathrm{Tl}_{0.03}$ films both near electrically isolated normal-metal ground planes and on clean fire-polished glass. Transition temperatures, obtained by fitting the data with the two-dimensional Aslamazov-Larkin theory of fluctuation conductance, were found to be significantly enhanced for films deposited over ground planes versus those deposited onto insulating substrates. Conductivity enhancement due to superconducting fluctuations was found to be much less than expected for the thinnest samples. This suppression was coincident with broadened superconductor transitions that are consistent with nonuniform sample thickness. Sufficiently thick films showed reasonable agreement with both the fluctuation theory and assumption of uniformity. We discuss discrepancies between our fits and the theory within the context of film morphology.
\end{abstract}

DOI: $10.1103 /$ PhysRevB.68.134502

PACS number(s): 74.78. $-\mathrm{w}, 74.40 .+\mathrm{k}, 74.62 .-\mathrm{c}$

The technique of quench condensation-evaporation onto cryogenically cooled substrates - has been used for decades to study metastable disorder in a variety of thin-film systems. ${ }^{1}$ Investigations of superconducting films produced with this technique have provided particularly fruitful insight into the basic mechanisms of superconductivity. The superconductor-insulator transition (SIT) is an example of a widely studied phenomenon utilizing quench condensation. ${ }^{2-19}$ In this transition, films evaporated just past the onset of a measurable conductance are found to show insulator properties: i. e., their resistance increases with decreasing temperature and extrapolates to $\infty$ at $T=0$. As the samples are incrementally grown to increased thicknesses, the resistance versus temperature, $R(T)$, behavior begins to resemble that of a superconductor. Depending on their morphology, films that have just crossed into the superconducting regime typically exhibit either suppressed transition temperatures $\left(T_{C}\right)$ or broadened resistive transitions.

It is possible to understand the SIT in these samples within the simple framework of the superconductor pair wave function: $\Psi_{S} \approx \Delta^{1 / 2} e^{i \phi}$, with amplitude $\Delta^{1 / 2}$ and phase $\phi . \Psi_{S}$ can be destroyed either by breaking long-range phase coherence or suppression of the amplitude. When samples are prepared by quench condensation onto insulating substrates, they generally appear to be granular in that they typically do not have a measurable conductance until tens of atomic layers of material are deposited..$^{1-10,20}$ Additionally, the presence of nearly bulk values for $T_{C}$ and the observation of an energy gap $(\Delta)$, even on the insulating side of the SIT, indicate that transport is dominated by superconductivity determined by the stability of phase between grains and intergrain hopping or tunneling. ${ }^{6}$ The broadened transitions observed in these samples are explained as phase breaking between grains or clusters of grains due to phase fluctuations. Samples that are quench condensed over $\mathrm{Ge}$ or $\mathrm{Sb}$ layers have been interpreted as having a "uniform" morphology. ${ }^{11-18}$ In these films, the onset of measurable conductance occurs at a thickness equivalent to only one or two atomic layers. The observed suppression of $T_{C}$ and $\Delta$ on the superconducting side of the SIT suggests that reduced amplitude and not phase variations drive the SIT.
SIT's have been observed in other systems as well. In particular, Josephson junction arrays can exhibit a SIT when placed in proximity to a two-dimensional (2D) electron gas that acts as a tunable dissipative medium. ${ }^{21}$ This behavior might be evidence for damping of fluctuations in the superconducting system by the normal resistance of the coupled medium. Other studies have involved measurements of superconducting samples that were in close proximity to normal-metal ground planes. In one case, results were interpreted as being consistent with Josephson array results. ${ }^{22}$ In other work, a $T_{C}$ enhancement attributed to Coulomb screening was observed, but no fluctuation damping was detected. ${ }^{18,23}$

The experiments reported here are the results of an attempt to detect changes in superconducting fluctuations in thin $\mathrm{Bi}$ films due to the presence of a nearby dissipative medium (a normal-metal ground plane). What distinguishes these measurements from most of the previous work is that we do not use a $\mathrm{Ge}$ or $\mathrm{Sb}$ layer under the $\mathrm{Bi}$ film. The motivation for our approach was threefold: first, $\mathrm{Ge}$ or $\mathrm{Sb}$ underlayers are known to effect the transport and superconducting properties of samples; ${ }^{16}$ second, these layers would serve to further isolate the sample from the normal-metal ground plane; and third, $\mathrm{Bi}$ films quenched condensed directly onto clean insulating substrates have been used to both motivate $^{24}$ and then ultimately support ${ }^{25-28}$ the fluctuation conductance theory of Aslamazov and Larkin. ${ }^{29}$ Our approach was simply to measure and analyze the resistive transitions of a series of $\mathrm{Bi}$ films that were deposited onto either clean glass substrates or over well-passivated $\mathrm{Al} / \mathrm{Al}_{2} \mathrm{O}_{3}$ ground planes.

Glass substrates were cleaned and fire polished before $\mathrm{Al}$ ground planes were deposited with the substrate held at room temperature. Ground planes ranged in thickness from 3 to 50 $\mathrm{nm}$ as measured by a quartz crystal microbalance. After deposition, the ground planes were allowed to oxidize in ambient air for 1-5 days. At that point Ag leads were deposited onto the substrate and over the ground plane as shown in Fig. 1. The substrates were then immediately mounted in a quench-evaporator cryostat and pumped with a diffusion pump before being cooled to $4 \mathrm{~K}$. Room-temperature two- 


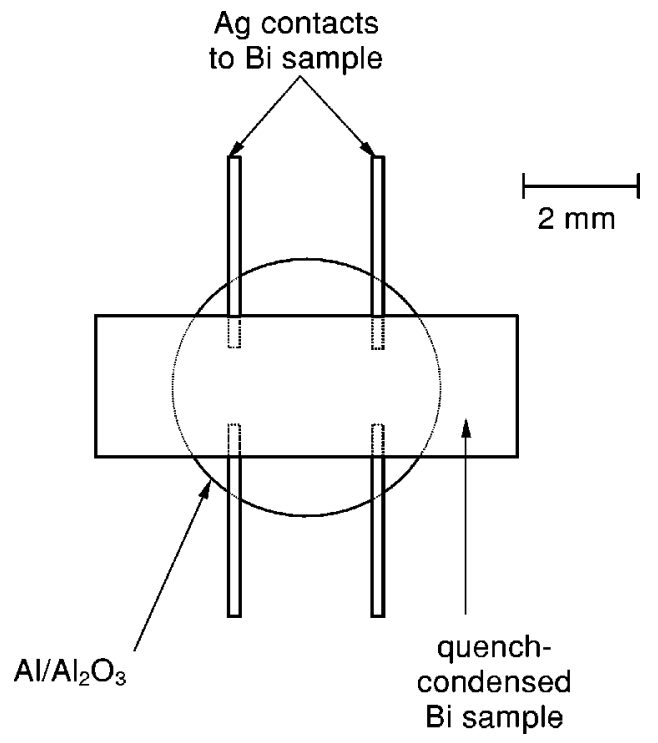

FIG. 1. Sample layout showing the $\mathrm{Ag}$ contacts for the four-wire Bi film measurement over a circular $\mathrm{Al} / \mathrm{Al}_{2} \mathrm{O}_{3}$ ground plane. The contact layout is the same when no ground plane is used.

wire resistance measurements between $\mathrm{Ag}$ leads over the ground planes were typically greater than $10^{8} \Omega$. At cryogenic temperatures the two-wire resistances exceeded the input impedance of our voltage amplifiers $\left(10^{10} \Omega\right)$. Since these values are many orders of magnitude higher than our sample resistance of interest, we expect no appreciable errors from the parallel conduction path through the ground plane. For samples without ground planes Ag leads were simply deposited onto clean glass and the same procedure as above followed.

Once the cryostat was cooled to liquid helium temperatures, $\mathrm{Bi}_{0.97} \mathrm{Tl}_{0.03}$ films were deposited onto the substrates held at $10 \mathrm{~K}$. This temperature insures that measurements during deposition are of normal-state $\mathrm{Bi}$. In addition, the evaporation chamber is completely submerged in liquid helium to produce ultra-high-vacuum conditions for the entire experimental run. The 3 at. \% $\mathrm{Tl}$ was used to stabilize the amorphous phase of $\mathrm{Bi}^{24}$ Since the focus of this investigation was on films on the superconducting side of the SIT, the initial deposition was stopped once the measurable resistance of the film was below $50 \mathrm{k} \Omega$. This resistance occurs at an approximate thickness of $2 \mathrm{~nm}$ in agreement with previous work. ${ }^{20}$ This average thickness is much greater than that observed for samples deposited onto $\mathrm{Ge}$ or $\mathrm{Sb},{ }^{12-15}$ indicating some inhomogeneity in the films. Also the onset of measurable conductance occurs more abruptly, suggesting the presence of clumps of material before the sample becomes electrically continuous. After deposition, $R(T)$ was measured using a four-wire lock-in amplifier technique for both the film and a $\mathrm{RuO}$ resistance thermometer and plotted directly on an analog $x-y$ plotter. Temperature was controlled using a small current source and a resistance heater mounted to the sample block. After each $R(T)$ curve was generated, additional material was deposited and the procedure was repeated.

Table I presents a summary of the nine experimental runs
TABLE I. Summary of parameters for the nine experimental runs presented in this work. Open symbols denote samples prepared onto clean glass substrates $(\mathrm{G} 1-\mathrm{G} 4)$, and solid symbols indicate those deposited onto ground planes (GP1-GP5). These symbols are used consistently in the figures that follow. The range of values for the fitted normal resistance per square, $R_{\square N}$, for each run is given in addition to the number of films measured. For the samples deposited onto ground planes, the thickness of the $\mathrm{Al}$ and the oxidation times are shown.

\begin{tabular}{cccccc}
\hline \hline Run & Symbol & $\begin{array}{c}\text { Range } \\
\text { of normal } \\
\text { resistance }(\Omega)\end{array}$ & $\begin{array}{c}\text { Number of } \\
\text { films }\end{array}$ & $\begin{array}{c}\text { Ground } \\
\text { plane } \\
\text { thickness } \\
(\mathrm{nm})\end{array}$ & $\begin{array}{c}\text { Oxidation } \\
\text { time } \\
\text { (days) }\end{array}$ \\
\hline G1 & $\bigcirc$ & $27.3-2914$ & 10 & & \\
G2 & $\nabla$ & $22.4-3086$ & 11 & & \\
G3 & $\square$ & $40.0-1411$ & 9 & & \\
G4 & $\diamond$ & $75.0-606$ & 6 & & \\
GP1 & - & $50.9-2491$ & 12 & 12 & 5 \\
GP2 & $\nabla$ & $46.4-10537$ & 14 & 24 & 2 \\
GP3 & $\square$ & $42.5-1954$ & 13 & 10 & 2 \\
GP4 & $\nabla$ & $48.2-711$ & 10 & 50 & 1 \\
GP5 & $\boldsymbol{\Delta}$ & $36.4-3506$ & 12 & 3 & 3 \\
\hline \hline
\end{tabular}

reported in this work, including details regarding the preparation of the ground planes. The symbols noted in the table are used consistently throughout the subsequent figures. Figure 2 shows typical results for a series of Bi films (G2) on a clean glass substrate. Note that the $T_{C}$ increases as thickness increases and normal state-resistance per square $\left(R_{\square N}\right)$ decreases. The solid curves were generated from our threeparameter fits to the Aslamazov-Larkin theory of paraconductance (details below). For even the highest-resistance

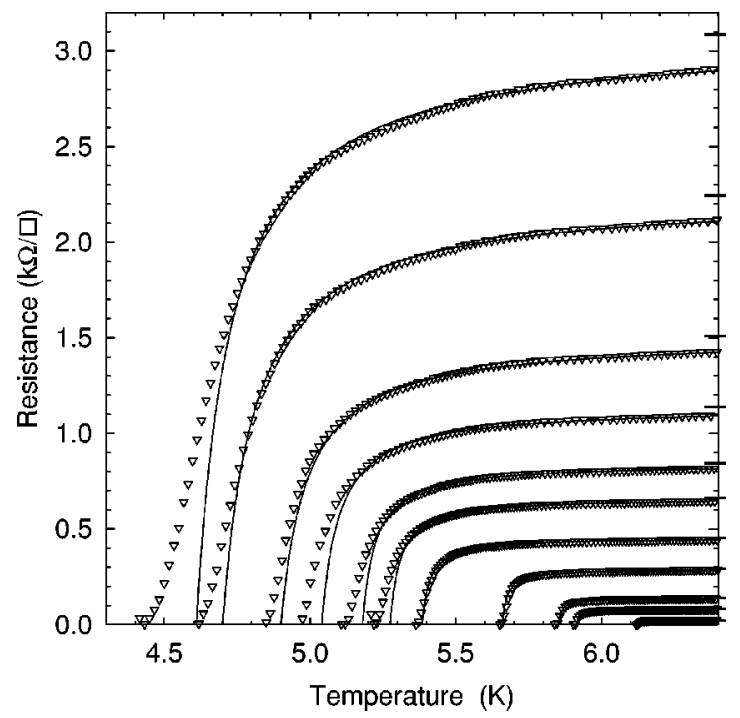

FIG. 2. Resistance vs temperature for run G2, an incrementally deposited series of quench-condensed Bi films on clean glass. As thickness increases, the normal-state resistance per square $\left(R_{\square N}\right)$ decreases. The solid curves are fits to Aslamazov-Larkin fluctuation theory (see text). The emphasized tick marks on the right axis indicate the $R_{\square N}$ values obtained from the fits to each curve. 

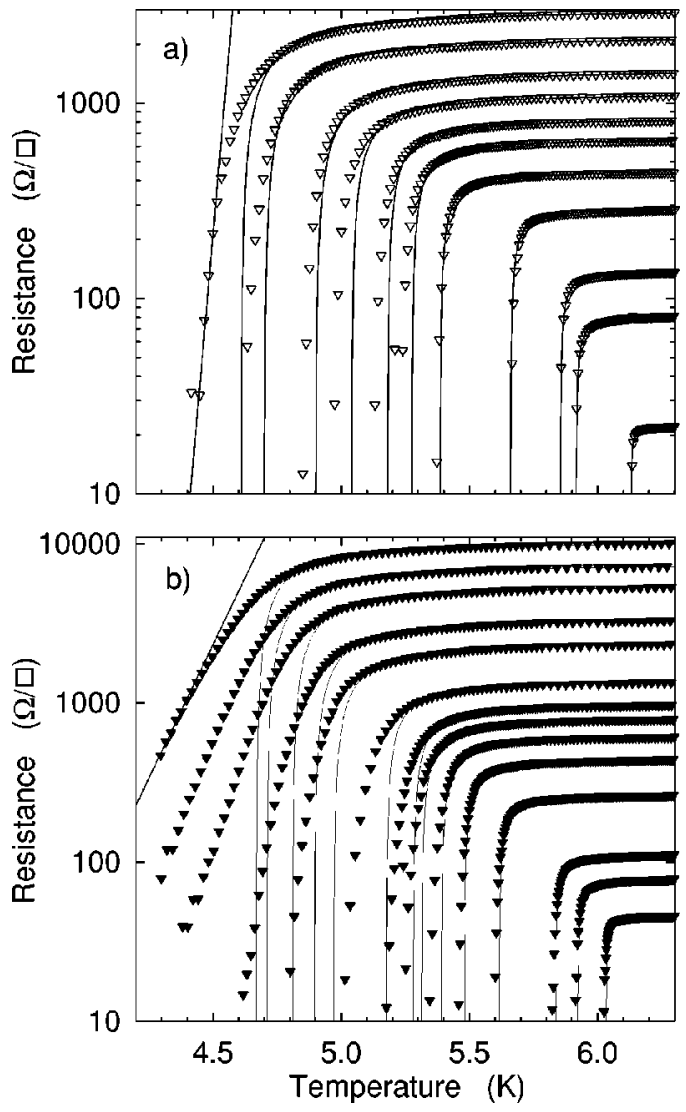

FIG. 3. (a) $R(T)$ for the same data as in Fig. 2 (run G2), plotted as $\log _{10}(R)$. (b) $R(T)$ data for run GP2, samples deposited over a normal ground plane. The preparation details of the ground plane are given in Table I. Solid curves are the Aslamazov-Larkin fits, except for the leftmost linear fits to the resistive tails (see text).

films, these fits agree well with the data down to about $1 / 2 R_{\square N}$. One of the fit parameters $R_{\square N}$ is noted for each curve as a large tick mark on the right axis of the plot.

Figure 3(a) shows the same data as Fig. 2 (G2) but now plotted as $\log _{10}(R)$ vs $T$, and Fig. 3(b) shows different results for a film over an $\mathrm{Al}$ ground plane that was oxidized for 2 days (GP2). Note that with the $\log _{10}(R)$ vs $T$ plots, the transition curves are easier to resolve for the entire range of measurements. The fits also work well down to about $1 / 2 R_{\square N}$ for the ground plane samples, and they clearly agree very well for the thickest samples in both plots. The leftmost linear fits to the resistive tails indicate an exponential dependence of resistance on temperature (see discussion below).

We have analyzed our results using the Aslamazov-Larkin theory of paraconductance as a starting point. Based on experimental work by Glover, ${ }^{24}$ Aslamazov and Larkin ${ }^{29}$ (AL) showed that for a superconductor of thickness $d$ and superconducting coherence length $\xi$, if $d \ll \xi$, the 2D paraconductance for a square of film $G_{\square}^{\prime}$ is given by

$$
G_{\square}^{\prime}=\frac{g_{\mathrm{AL}}}{t},
$$

where

$$
g_{\mathrm{AL}} \equiv \frac{e^{2}}{16 \hbar} \text { and } t \equiv \frac{T-T_{C}}{T_{C}} .
$$

Together with the normal conductance per square $G_{\square N}$ this predicts that

$$
R_{\square}(T)=\frac{1}{G_{\square N}+G_{\square}^{\prime}}
$$

or

$$
\frac{1}{R_{\square}(T)}=G_{\square N}+G_{\square}^{\prime}=\frac{1}{R_{\square N}}+\frac{g_{\mathrm{AL}}}{t} .
$$

Rearranging we have

$$
\frac{1}{R_{\square}(T)}-\frac{1}{R_{\square N}}=\frac{R_{\square N}-R_{\square}(T)}{R_{\square N} R_{\square}(T)}=\frac{g_{\mathrm{AL}}}{t}
$$

or finally

$$
r=\frac{t}{g_{\mathrm{AL}} R_{\square N}},
$$

with

$$
r \equiv \frac{R_{\square}(T)}{R_{\square N}-R_{\square}(T)}=\frac{R(T)}{R_{N}-R(T)} .
$$

This form of $r(t)$ gives a more convenient dimensionless version of the AL theory.

In order to fit our experimental results we used three fitting parameters: $R_{N}, T_{C}$, and $g_{\text {expt }}$. Note that we have still assumed $r=\alpha t$ in the absence of any detailed treatment of other effects. In this form, calculating $r$ does not require knowledge of the number of squares of the sample in order to fit our results. The number of squares is only important for retrieving a value of $g_{\text {expt }}=1 / \alpha R_{\square N}$. We also point out that although $R_{N}$ and $T_{C}$ are fitting parameters, they are constrained by physical reality. We made some attempt at measuring $R_{N}$ by finding the film resistance at about $12 \mathrm{~K}(2-3$ times $T_{C}$ ). However, within the framework of the AL theory, high-resistance films will have non-negligible fluctuation conductance contributions at even higher temperatures. This fact makes our measured $R_{N}$ results useful only as starting values in the fitting process. The discrepancy is apparent in Fig. 2 where the $R_{N}$ values are marked on the right axis. In practice, linear fits were performed for both linear $r$ vs $t$ representations and $\log _{10}(r)$ vs $\log _{10}(t)$ representations of the data. In the latter case, the fitted slopes were between 0.997 and 1.003, indicating very linear behavior. Figure 4 shows a set of fits for the data from Fig. 3(b) and $R$ $>3 / 4 R_{N}$ plotted on a log-log scale.

It is important to emphasize the high sensitivity of our fits to small changes in the parameters $R_{N}$ and $T_{C}$. We found that for small variations in $R_{N}$, the high- $r$ region of the curve became very nonlinear, whereas changes in $T_{C}$ caused the low- $r$ region to deviate. Changes in either parameter of more than $1 \%$ or $2 \%$ from the best-fit values typically resulted in curves that could not reasonably be described as linear. 


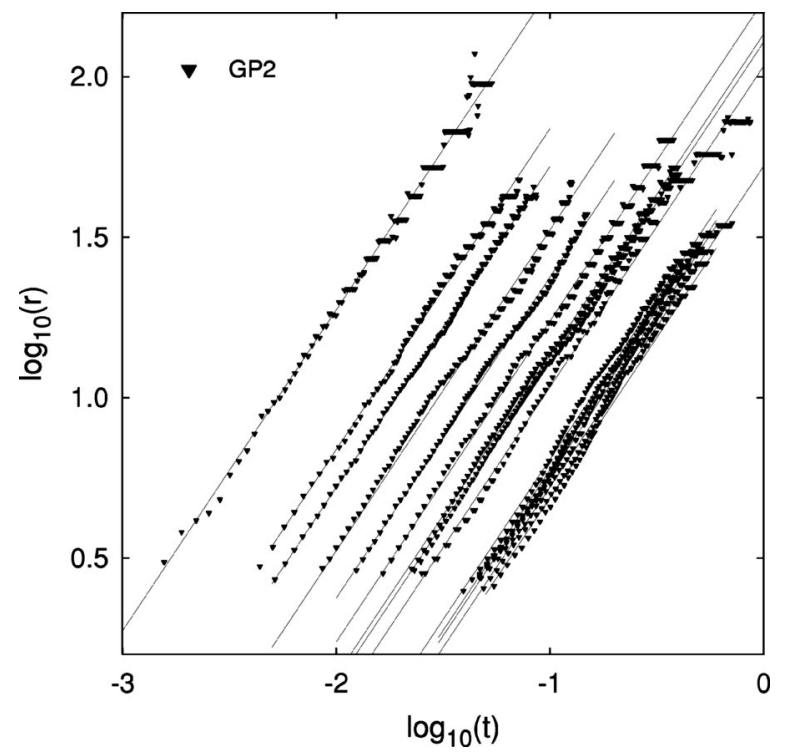

FIG. 4. $\log _{10}(r)$ vs $\log _{10}(t)$ for the data in Fig. 3(b) (run GP2). $r=R(T) /\left[R_{N}-R(T)\right]$ and $t=\left(T-T_{C}\right) / T_{C}$. The straight lines have slope 1.00 and are calculated from fits to $r=t / g_{\text {expt }} R_{N}$, a dimensionless version of the Aslamazov-Larkin form.

Results of fits for four series of films on clean glass (open symbols) and five series over ground planes (solid symbols) are summarized in Fig. 5. We have plotted both the fitted $T_{C}$ and $g_{\text {expt }}$ values as a function of our fitted $R_{\square N}$ results. Note that the abscissa is the same for both plots, so that $T_{C}$ values lie vertically above their corresponding $g_{\text {expt }}$. On the $T_{C}$ plot we have also included data for quench-condensed $\mathrm{Bi}$ on $\mathrm{Ge}$ underlayers for comparison. ${ }^{14}$ On the $g_{\text {expt }}$ plots we have scaled our fitted values to the theoretical $e^{2} / 16 \hbar=1.52$ $\times 10^{-5} \Omega^{-1}$.

Two main features are apparent in these data. First, given a particular $R_{\square N}$ the $T_{C}$ 's of our films are much higher than those of the "uniform" Bi over Ge samples. Second, for higher $R_{\square N}$ values the fitted $g_{\text {expt }}$ results are clearly lower than the theoretical value and they follow a downward trend as $R_{\square N}$ increases. As for the comparison between samples over ground planes and those on clean glass, the $T_{C}$ 's are enhanced over ground planes in all but one of the experimental runs.

Early work on paraconductance found excellent agreement between the theoretical value $g_{\mathrm{AL}}$ and the experimental value $g_{\text {expt }}$ when the latter was averaged over several samples. ${ }^{25-27}$ Furthermore, there was no evidence of a dependence of $g_{\text {expt }}$ on film resistance. However, the agreement was found for samples with a reported normal-state resistance of roughly $300 \Omega$ and less. To explain the discrepancies that were observed, variations in sample morphology were suggested as a likely cause. ${ }^{26,30}$ In Fig. 5 it is evident that the $T_{C}$ results become consistent with the $T_{C}$ values of "uniform" films at a sheet resistance around 200-300 $\Omega$. It is also true that despite the scatter in our $g_{\text {expt }}$ values, the average for all films below $300 \Omega$ sheet resistance is 1.57 $\pm 0.37 \times 10^{-5} \Omega^{-1}$, close to the theoretical value.

The explanation of our results focuses on the discrepancies between the data and fitted curves. The fits were gener-

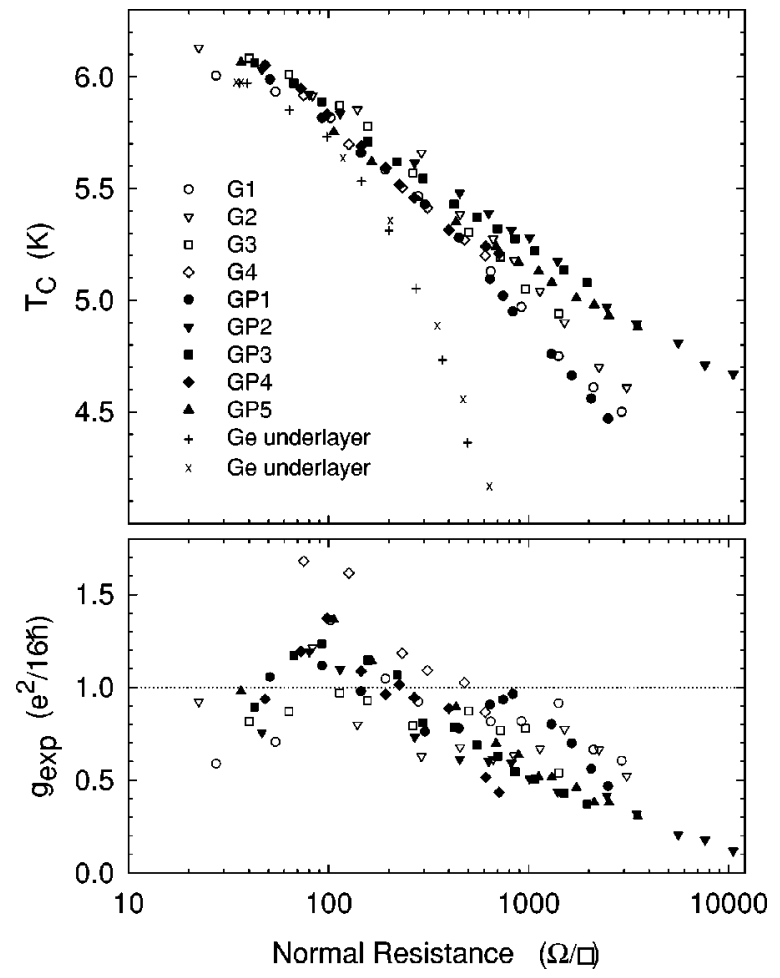

FIG. 5. Parameters for all samples from fits like those in Fig. 4. Plotted are $T_{C}$ and $g_{\text {expt }}$ vs $R_{\square N}$. Open symbols are for Bi on clean glass $(\mathrm{G} 1-\mathrm{G} 4)$ and solid symbols are for $\mathrm{Bi}$ over ground planes (GP1-GP5). The preparation details of the ground planes are given in Table I. The + and $\times$ data are for "uniform" Bi deposited onto Ge-coated substrates (Ref. 14).

ated using the $R>3 / 4 R_{N}$ portion of the $R(T)$ data. For samples with $R_{\square N}<300 \Omega$, there is excellent agreement between the fits and the measurements even below our $3 / 4 R_{N}$ cutoff. The predicted curves deviate more significantly from the $R<3 / 4 R_{N}$ data for the higher resistance samples. We also note that for samples with $R_{\square N}>300 \Omega$ this deviation coincides with $R(T)$ curves exhibiting the broadened exponential tail typically seen in granular films. This behavior is most apparent in the log-linear plots of Fig. 3, where the low-resistance behavior appears linear. In Fig. 6 we estimate a transition width $T_{0}$ where $R \sim e^{T / T_{0}}$ for all films over 300 $\Omega$. Despite significant scatter in our results (our focus during measurements was on fluctuations and therefore the high resistance end of the transitions), it appears that transition widths are determined by $R_{\square N}$. This conclusion is consistent with results for granular $\mathrm{Pb}, \mathrm{Pb}-\mathrm{Ag}$, and $\mathrm{Sn}$ systems, ${ }^{10}$ however, our transitions are narrower and apparently do not follow the same universal width. An explanation for this discrepancy is that since $\mathrm{Bi}$ is a superconductor only in its amorphous phase, a granular morphology typical of crystalline grains observed in $\mathrm{Pb}$ (Ref. 8) and other systems is perhaps not the most appropriate description. Figure 7 shows a simple illustration that summarizes our results. We have plotted $R(T)$ for two films: one is over a ground plane and one is on clean glass. They have roughly the same normal-state resistance, but the film over a ground plane has a significantly higher $T_{C}$. Also note that the transition width as given 


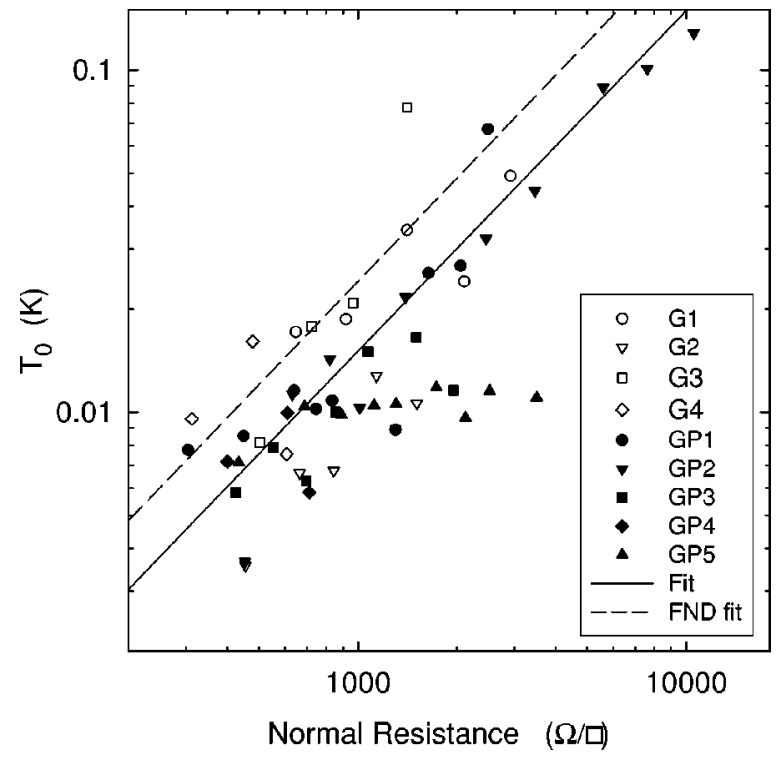

FIG. 6. Compilation of $T_{0}$ values for all films with $R_{\square N}$ greater than $300 \Omega$ using the assumption that $R(T) \approx e^{T / T_{0}}$ in the lowtemperature end of the resistive transitions. A linear fit is shown and comparison with the linear fit from Frydman et al. (Ref. 10).

by $T_{0}$ is roughly the same for both films. Finally, it is interesting to note that tunneling studies of granular $\mathrm{Pb}$ in perpendicular magnetic fields found normal cores appearing at a very similar normal-state resistance of $375 \Omega .{ }^{7}$ This resistance corresponds to a thickness where field lines begin to penetrate the film, not just the empty regions between the grains.

In order to differentiate our language from "granular"

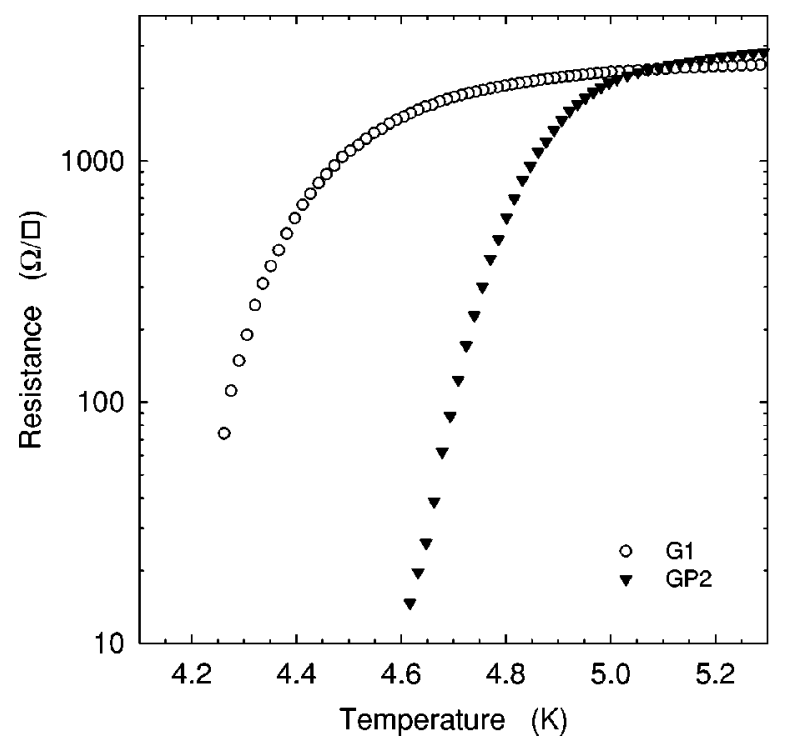

FIG. 7. Comparison of two different $R(T)$ curves with similar normal-state resistance. The open symbols are for a film on clean glass (G1), and the solid symbols are for a film over a ground plane (GP2). Note that on this $\log _{10}(R)$ vs $T$ scale, the low-temperature ends of the transitions are roughly linear, and the slopes are about the same. This trend indicates an $R(T) \approx e^{T / T_{0}}$ dependence where $T_{0}$ depends only on $R_{\square N}$. which tends to imply crystalline grains, we chose the term "undulant" to mean nonuniform thickness. It is likely that this morphology reflects the fact that films grow first as isolated clumps of material. ${ }^{20}$ It is unclear whether the films continue to look like isolated amorphous clumps or simply a slab that is thicker in some regions than in others. Either structure would give rise to spatial variations in $T_{C}$ (Ref. 30) and therefore an "effective granularity" to our samples. Since thicker films eventually agree well with predictions for uniform thickness, the thickness variation picture is perhaps more straightforward. Given the significant suppression of $T_{C}$ below the bulk value $(\sim 6 \mathrm{~K})$ in our samples, small incremental increases in thickness will give rise to significant changes in $T_{C}$. Furthermore, small variations in thickness from place to place would lead to spatially varying $T_{C}$ 's in either case. We are aware of one other "granular" material, $\mathrm{Ga}$, which prepared on a clean insulating substrate also showed significant changes in the $T_{C} \cdot{ }^{3}$ It is interesting that the $\mathrm{Ga}$ films in these experiments were also amorphous.

An undulant morphology also explains the higher $T_{C}$ 's for given $R_{\square N}$ with respect to the uniform case. If the sample has thicker regions of higher $T_{C}$, these regions are coupled by the thinner, lower $T_{C}$ regions which behave as normal metal. Extending this assumption to our ground plane versus clean substrate samples, we conclude that films grown over ground planes have more thickness variation. This morphological difference leads to higher normal-state resistance (thinner connecting regions) and higher $T_{C}$. This result is also consistent with other work which did use a Ge underlayer and found both $T_{C}$ and $R_{\square N}$ higher over ground planes compared to the same sample over a clean insulating substrate. ${ }^{18}$ A morphological difference might be an alternate explanation for those results. We also performed additional measurements on samples that had one region over a ground plane and one region not. In these samples it was found that ground plane films did have higher normal resistance for the same thickness. Coupled with our current analysis, we are reasonably confident that the differences indicate a more undulant morphology over the ground plane than on the clean substrate. One explanation for this difference might be the nonuniform nature of the $\mathrm{Al}$ films grown on roomtemperature substrates and the resultant $\mathrm{Al}_{2} \mathrm{O}_{3}$. However, it should be noted that structural variations due to different substrates have not been observed in STM studies of granular quench-condensed films. ${ }^{8}$

In order to further understand the apparent reduced $g_{\text {expt }}$ values, increased $T_{C}$ 's and the difference between the ground plane and clean glass samples within the context of our model, we have plotted our data from Fig. 5 using a rescaled resistance axis in Fig. 8. It is important to point out that the $T_{C}$ of the thicker regions of the film will dominate the onset of the resistive transition (thinner regions would still be normal) and therefore the calculated $g_{\text {expt }}$ value. However, the thinner regions of the films dominate the $R_{\square N}$ value. Since knowing $G_{\square N}\left(1 / R_{\square N}\right)$ is critical to calculating the fluctuation conductance, the fact that we are using $R_{\square N}$ values that are higher than the relevant value (the $R_{\square N}$ of the thicker region would be lower) is causing our $g_{\text {expt }}=1 / \alpha R_{\square N}$ result to be reduced. By rescaling our resistance axis using $R_{\text {eff }}$ 


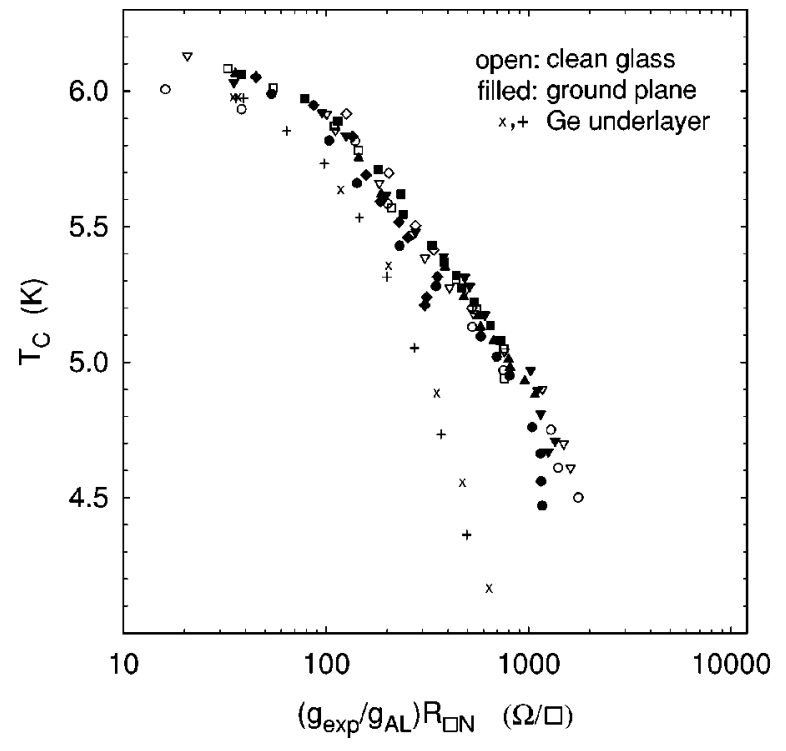

FIG. 8. $T_{C}$ vs $R$ data from Fig. 5(a) with the resistance axis rescaled as $R=\left(g_{\text {expt }} / g_{\mathrm{AL}}\right) R_{\square N}$. The theoretical value for $2 \mathrm{D}$ films is given by $g_{\mathrm{AL}}=e^{2} / 16 \hbar . T_{C}$ vs $R_{\square N}$ is also plotted (and not scaled) for $\mathrm{Bi}$ deposited onto Ge underlayers (Ref. 14). Note that although the present data do not fall exactly on that of the "uniform" films, the nonuniform data collapse onto the same curve and resemble that of the "uniform" samples. This result suggests that we can define an "effective" resistance as probed by the length scales of superconducting fluctuations. The shorter morphological length scales control the measured normal resistance.

$=\left(g_{\text {expt }} / g_{\mathrm{AL}}\right) R_{\square N}$ we are essentially finding the normal resistance for which the $g_{\text {expt }}$ would equal the theoretical value. When rescaled this way, our data collapse onto a single curve. This curve also follows the trend of the "uniform" $\mathrm{Bi}$ on Ge samples. The success of this rescaling approach suggests that our undulant film model accounts for the effects that we have observed.

Because of the morphology-dominated behavior in our samples, it is not possible to comment on screening or damping effects. It is also important to consider how much morphological issues of this kind might impact interpretation of other work. Although the majority of these other experiments have been performed on $\mathrm{Bi} / \mathrm{Ge}$ samples, the much smaller thickness of the samples could lead to small variations producing rather larger effects in the measured $T_{C}$. Of greatest concern is the significant difference between clean glass and $\mathrm{Al} / \mathrm{Al}_{2} \mathrm{O}_{3}$. If different substrate treatment can cause enough morphological variation to locally change the sample $T_{C}$ by up to $0.5 \mathrm{~K}$, it is important to rule out these effects.

Previous authors have concluded that there is some granularity present even in samples with Ge underlayers. ${ }^{19}$ This conclusion was partially supported by using reflection high energy electron diffraction (RHEED) to discern 5-nm clumps in quench-condensed $\mathrm{Bi}$ films. This value is consistent with earlier work that found the critical average thickness for conduction of $\mathrm{Bi}$ to be approximately 2-3 nm. ${ }^{20}$ Hysteretic current-voltage curves were also cited ${ }^{19}$ as indicating granularity in $\mathrm{Bi}$ samples, although these curves are consistent with sample heating (power $\gg 10 \mu \mathrm{W}$ ). Finally, these samples were prepared on Ge that was exposed to air before the cooldown. Some nonuniformity might result from that treatment.

In conclusion, we have measured quench-condensed $\mathrm{Bi}$ films without Ge underlayers. Like other systems deposited onto insulating substrates, there is clear evidence for nonuniform (undulant) thickness. Our data for the resistive transitions of our thinnest samples are consistent with those for granular superconducting films. Thick films exhibit transition curves that follow the standard Aslamazov-Larkin theory of paraconductance consistent with the earliest work on this subject. $^{25-28}$ Our analysis within the framework of this theory provides an approach at quantifying the transition between samples in the clearly undulant regime and those which can be considered as relatively uniform. Although we believe that we are seeing clear evidence of undulant morphology in our films, this question might also be worth investigating more closely in samples prepared over Ge or Sb.

We gratefully acknowledge valuable discussions with $\mathrm{R}$. Dynes, R. Glover, J. Valles, P. Xiong, P. Kesten, J. Birmingham, and W. DeHart and the invaluable technical support of S. Tharaud. This work was funded by Santa Clara University IBM Faculty Research Grants and gifts from T. Mahatdejkul and National Semiconductor Corporation.
*Current address: Department of Physics, University of California, Berkeley, CA 94720.

${ }^{1}$ W. Buckel and R. Hilsch, Z. Phys. 138, 109 (1954).

${ }^{2}$ R. C. Dynes, J. P. Garno, and J. M. Rowell, Phys. Rev. Lett. 40, 479 (1978).

${ }^{3}$ H. M. Jaeger, D. B. Haviland, B. G. Orr, and A. M. Goldman, Phys. Rev. B 40, 182 (1989), and references within.

${ }^{4}$ R. P. Barber, Jr. and R. E. Glover III, Phys. Rev. B 42, 6754 (1990)

${ }^{5}$ R. P. Barber, Jr. and R. C. Dynes, Phys. Rev. B 48, 10618 (1993).

${ }^{6}$ R. P. Barber, Jr., L. M. Merchant, A. La Porta, and R. C. Dynes, Phys. Rev. B 49, 3409 (1994).

${ }^{7}$ S. Y. Hsu and J. M. Valles, Jr., Phys. Rev. B 49, 6416 (1994).

${ }^{8}$ K. L. Ekinci and J. M. Valles, Jr., Phys. Rev. Lett. 82, 1518 (1999).
${ }^{9}$ S. R. Khan, E. M. Pedersen, B. Kain, A. J. Jordan, and R. P. Barber, Jr., Phys. Rev. B 61, 5909 (2000).

${ }^{10}$ A. Frydman, O. Naaman, and R. C. Dynes, Phys. Rev. B 66, 052509 (2002).

${ }^{11}$ M. Strongin, R. S. Thompson, O. F. Kammerer, and J. E. Crow, Phys. Rev. B 1, 1078 (1970).

${ }^{12}$ R. C. Dynes, A. E. White, J. M. Graybeal, and J. P. Garno, Phys. Rev. Lett. 57, 2195 (1986).

${ }^{13}$ D. B. Haviland, Y. Liu, and A. M. Goldman, Phys. Rev. Lett. 62, 2180 (1989).

${ }^{14}$ R. Barber, Jr., and R. E. Glover III, Bull. Am. Phys. Soc. 36 (3), 663 (1991).

${ }^{15}$ J. M. Valles, Jr., R. C. Dynes, and J. P. Garno, Phys. Rev. Lett. 69, 3567 (1992).

${ }^{16}$ P. Xiong, A. V. Herzog, and R. C. Dynes, Phys. Rev. B 52, 3795 
(1995).

${ }^{17}$ J. A. Chervenak and J. M. Valles, Jr., Phys. Rev. B 61, 9245 (2000).

${ }^{18}$ E. G. Astrakharchik and C. J. Adkins, J. Phys.: Condens. Matter 10, 4509 (1998).

${ }^{19}$ G. Sambandamurthy, K. Das Gupta, and N. Chandrasekhar, Solid State Commun. 118, 431 (2001); Phys. Rev. B 64, 014506 (2001).

${ }^{20}$ D. Gottschalk, R. Hilsch, and D. Korn, Z. Phys. 244, 245 (1971).

${ }^{21}$ A. J. Rimberg, T. R. Ho, C. Kurdak, J. Clarke, K. L. Chapman, and A. C. Gossard, Phys. Rev. Lett. 78, 2632 (1997).

${ }^{22}$ N. Mason and A. Kapitulnik, Phys. Rev. B 65, 220505 (2002).
${ }^{23}$ E. G. Astrakharchik and C. J. Adkins, Phys. Rev. B 50, 13622 (1994).

${ }^{24}$ R. E. Glover, Phys. Lett. 25A, 542 (1967).

${ }^{25}$ D. C. Naugle and R. E. Glover, Phys. Lett. 28A, 110 (1968).

${ }^{26}$ R. E. Glover III, in Progress in Low Temperature Physics, edited by F. Chilton (North-Holland, Amsterdam, 1970), Vol. 6, Chap. 7.

${ }^{27}$ R. E. Glover III, in Proceedings of the International Conference on the Science of Superconductivity, edited by F. Chilton (NorthHolland, Amsterdam, 1971), pp. 3-23.

${ }^{28}$ G. Bergmann, Phys. Rep. 27, 159 (1976).

${ }^{29}$ L. G. Aslamazov and A. I. Larkin, Phys. Lett. 26A, 238 (1968).

${ }^{30}$ R. E. Glover III, S. Moser, and F. Baumann, J. Low Temp. Phys. 5, 519 (1971). 